\title{
Assessment of current and future regional climate scenarios for Mexico
}

\author{
Víctor Magaña ${ }^{1, *}$, Cecilia Conde ${ }^{1}$, Oscar Sánchez ${ }^{1}$, Carlos Gay ${ }^{2}$ \\ ${ }^{1}$ Centro de Ciencias de la Atmósfera, Universidad Nacional Autónoma de México, Ciudad Universitaria, Circuito Exterior \\ 04510, México, DF México \\ ${ }^{2}$ Unidad de Cooperación y Convenios Internacionales, Instituto Nacional de Ecología, Avenida Revolución 1425 nivel 31 , \\ 01040, México, DF México
}

\begin{abstract}
Current climate and climate change scenarios are the basis for climate change vulnerability and adaptation studies. Comparison between the current situation and the one that would prevail under climate change conditions allows for the identification of vulnerable regions and the quantification of vulnerability. This may be done by evaluating the land area adversely affected by climate change (e.g. with reduction in agricultural yields or decrease in the level of water reservoirs). Specific regionalization criteria and methods based on physical considerations should be applied in the development of regional climate scenarios. This is the principle followed in studies using analogue scenarios. A methodology based on the direct interpolation of general circulation model (GCM) outputs to the study region under $1 \times \mathrm{CO}_{2}$ and $2 \times \mathrm{CO}_{2}$ conditions may be used as a first approach for the development of climate change scenarios. That methodology was applied in this study in order to be consistent with the assessments performed by more than 50 countries involved in the U.S. Country Studies Program and to allow for the comparison and integration of the results from several countries. However, an alternative methodology based on multiple regression was also applied by the Scenario task group of the Mexico Country Study. This methodology is considered to be more appropriate for regionalization purposes
\end{abstract}

KEY WORDS: Global climate change - General circulation models - Climate scenarios - Statistical models - Regionalization - Mexico Country Study

\section{INTRODUCTION}

The Mexico Country Study was initiated in 1994 and completed in 1995. It comprised 3 main sections: greenhouse gas emission inventory, scenarios, and vulnerability. This study, supported by the U.S. Country Studies Program, was an initiative of the Instituto Nacional de Ecología (INE) under the auspices of the Secretaría de Medio Ambiente, Recursos Naturales y Pesca (SEMARNAP) of the Mexican Government.

Seven sectors were addressed within the vulnerability section of the study: coastal areas; agriculture; hydrology; forests; desertification and droughts; human settlements; and energy and industry. Except

\footnotetext{
•E-mail: victor@regino.atmosfcu.unam.mx
}

for the coastal areas sector, all these required the development of climate scenarios as a basis for the vulnerability and adaptation analysis. Thus, both current climate and potential climate change scenarios were developed, mainly on the basis of general circulation model (GCM) simulations. This paper examines the adequacy of these scenarios for regional vulnerability analysis.

\section{METHODS}

Several criteria were adopted to determine the current climate scenario to be used, depending on the requirements of the particular vulnerability study being carried out. The type, availability, homogeneity and spatial scale of the data were some of the factors 


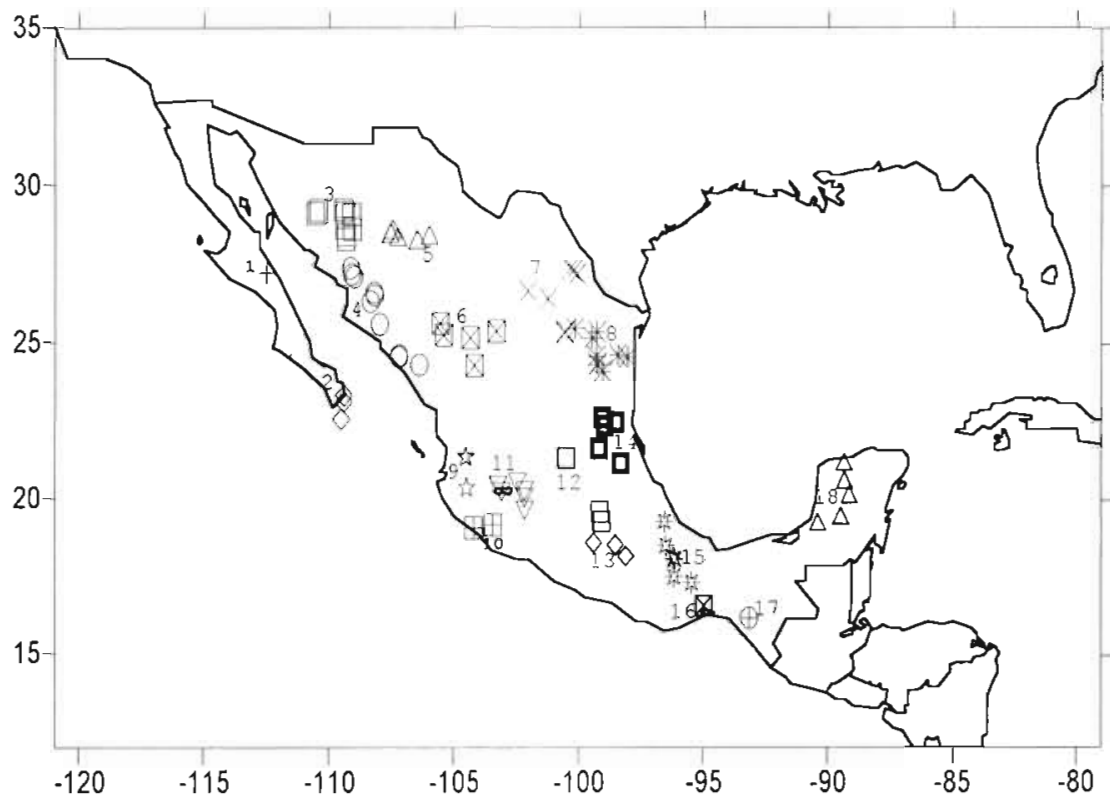

Fig. 1 The 18 climatic regions of Mexico proposed by Douglas. The symbols represent the weather stations for each Douglas region
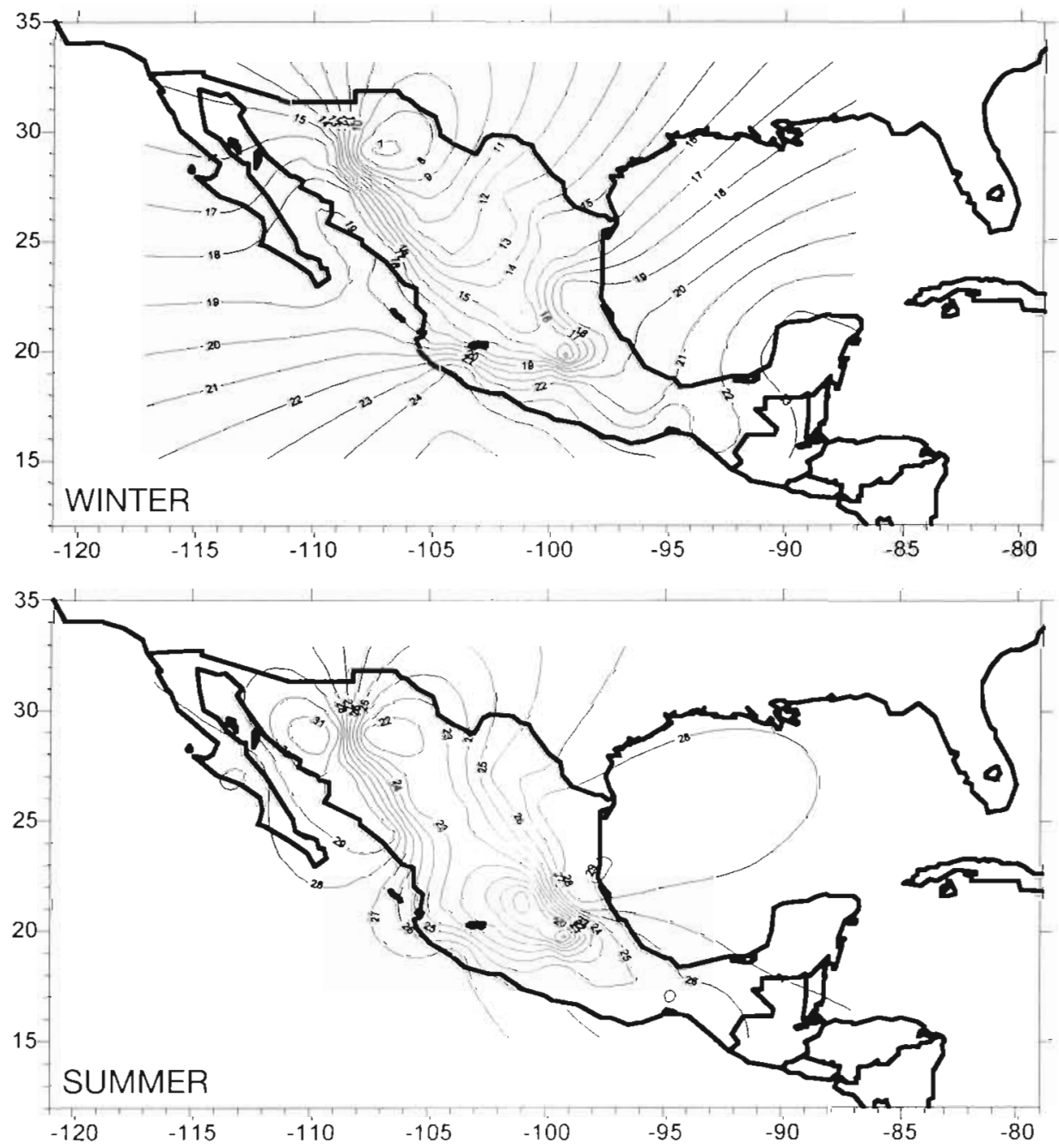

Fig. 2. Baseline temperature scenario (according to Douglas) in ${ }^{\circ} \mathrm{C}$ 

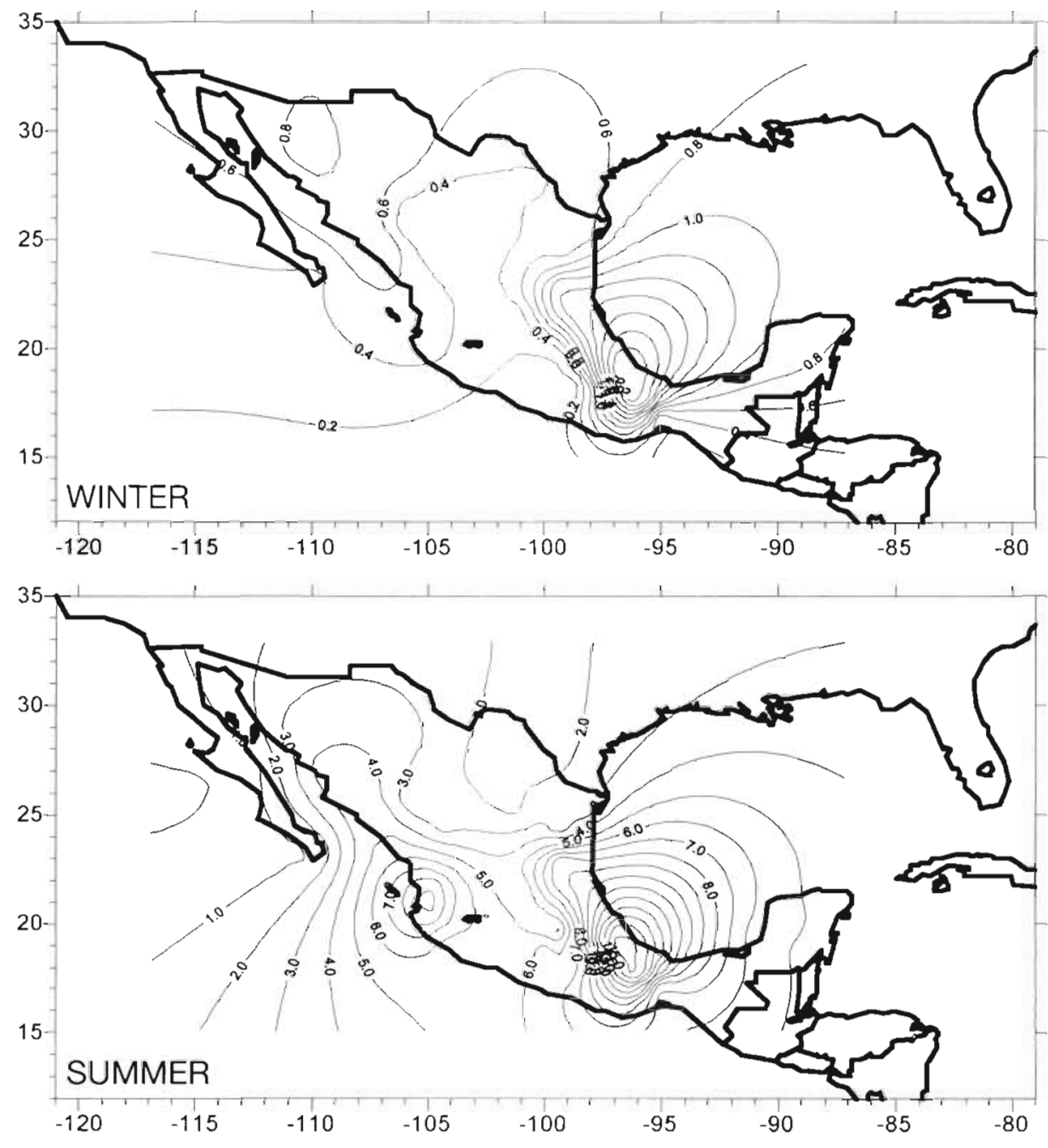

Fig. 3. Baseline precipitation scenario (according to Douglas) in $\mathrm{mm} \mathrm{d}^{-1}$

that were taken into account. Daily temperature (maximum and minimum values), precipitation and radiation data for specific sites were necessary for certain studies (e.g. for the application of the CERES wheat production model in agricultural studies). Mean monthly, seasonal and/or annual values of basic climatic variables (temperature and precipitation) were used in other cases. Data series for periods over $30 \mathrm{yr}$ and information on wind velocity (for desertification assessments) were also required in some cases. Daily and/or monthly values obtained from the Servicio Meteorológico Nacional (SMN) database and processed with the CLICOM system (a data management module used by SMN to handle surface weather station data) were also required for the conduct of certain assessments.

Temperature and precipitation databases for the current climate scenarios to be used in the vulnerability studies were produced in 2 stages. In the first stage (Conde et al. 1994), data from the 1941 to 1970 period for 23 points from a $2.5^{\circ} \times 2.5^{\circ}$ grid over Mexico, used by the Climate Thermodynamic Model (Adem 1982), were made available. Climate data for Mexico obtained from the CLIM database (ftp: ncardata. ucar.edu) used by NCAR (National Center for Atmospheric Research, Boulder, CO, USA) were also included.

In the second stage, data from the 1950 to 1980 period for 18 regions of the country (Conde et al. 1995), processed by Dr Arthur Douglas from the University of Creighton, Nebraska, USA (ftp.hurricane.ncdc.noaa. gov) were made available. The weather station records have been grouped by Douglas into 18 regions on the basis of the density, the altitude and the correlation among data. The results presented in this paper were produced on the basis of this methodology. 

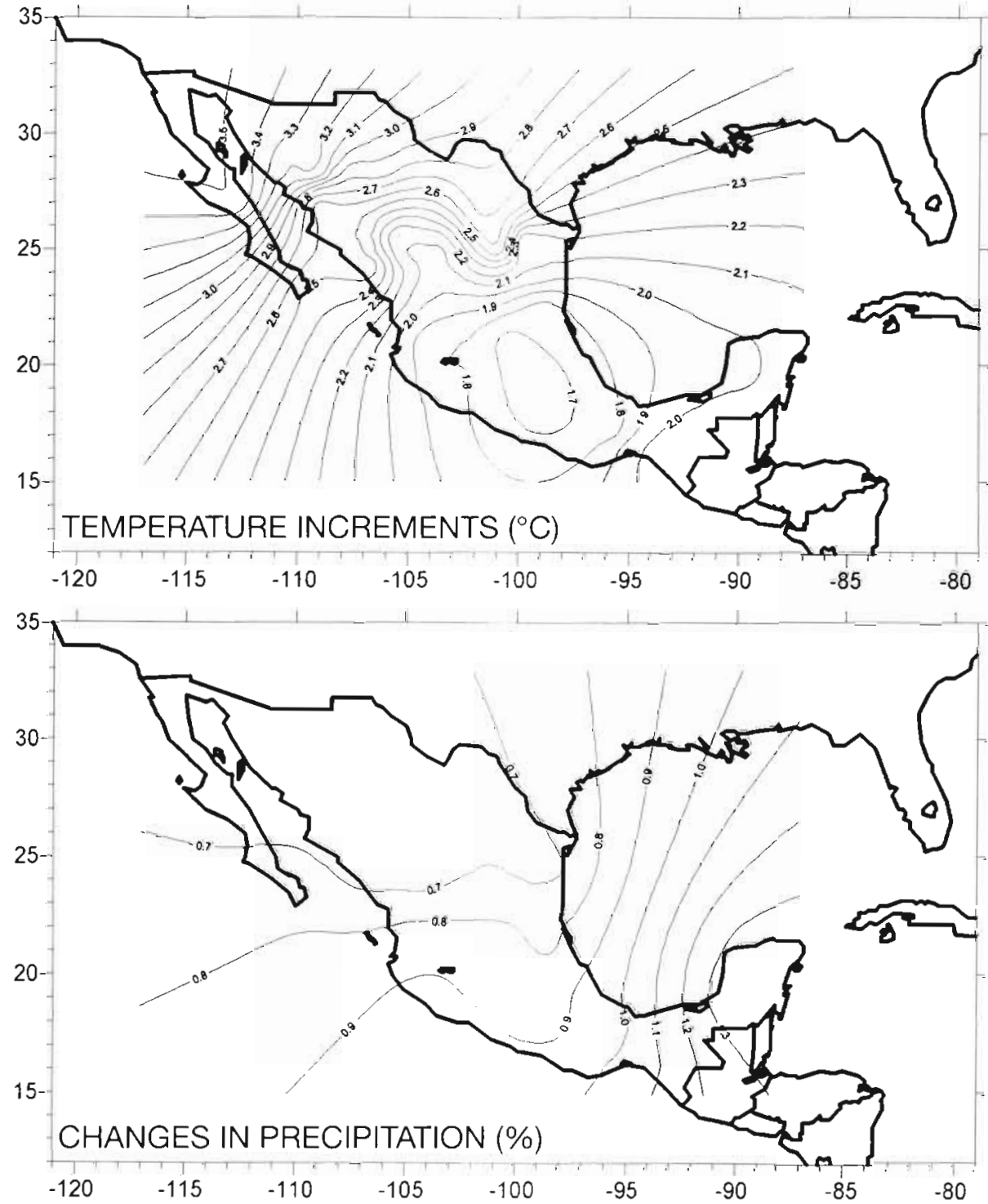

Fig. 4. CCCM-derived winter temperature and precipitation scenarios

Two methods were used for the development of climate change scenarios. The first one was based on arbitrary incremental changes in temperature $(+2$ and $+4^{\circ} \mathrm{C}$ ) and in precipitation ( $\pm 10 \%$ and $\pm 20 \%$ ) as well as combinations of both (e.g. $+2^{\circ} \mathrm{C}$ and $+20 \%$ ). The second one is based on changes in temperature, precipitation and radiation under a doubled atmospheric carbon dioxide condition $\left(2 \times \mathrm{CO}_{2}\right)$, as estimated from outputs of 2 GCMs. The models used were the GFDLR30 (Geophysical Fluid Dynamic Laboratory) and CCCM (Canadian Climate Centre Model). The variations derived from the GFDL-R30 transient model for the fourth, seventh and tenth decades were also analyzed.

This second method was applied with caution since, in spite of being the most widely used for the develop- ment of global climate change scenarios, direct interpolation of GCM results poses problems for the creation of regional and local scenarios.

The use of analogue scenarios and statistical models for correlating large-scale circulation patterns to local climate (Magaña 1994) was proposed as an alternative method to direct interpolation for the interpretation of the GCM outputs at a regional scale. Outputs from the coupled NCAR and GFDL models were used to analyze temperature and precipitation anomalies. The control (current) and doubled $\mathrm{CO}_{2}$ runs of the above models, obtained via the Internet (ftp.dkrz.de), were used to improve the regional assessment. A statistical analysis for assessing the consistency between the local variables (temperature and precipitation) and the observed large-scale variables was carried out. The 

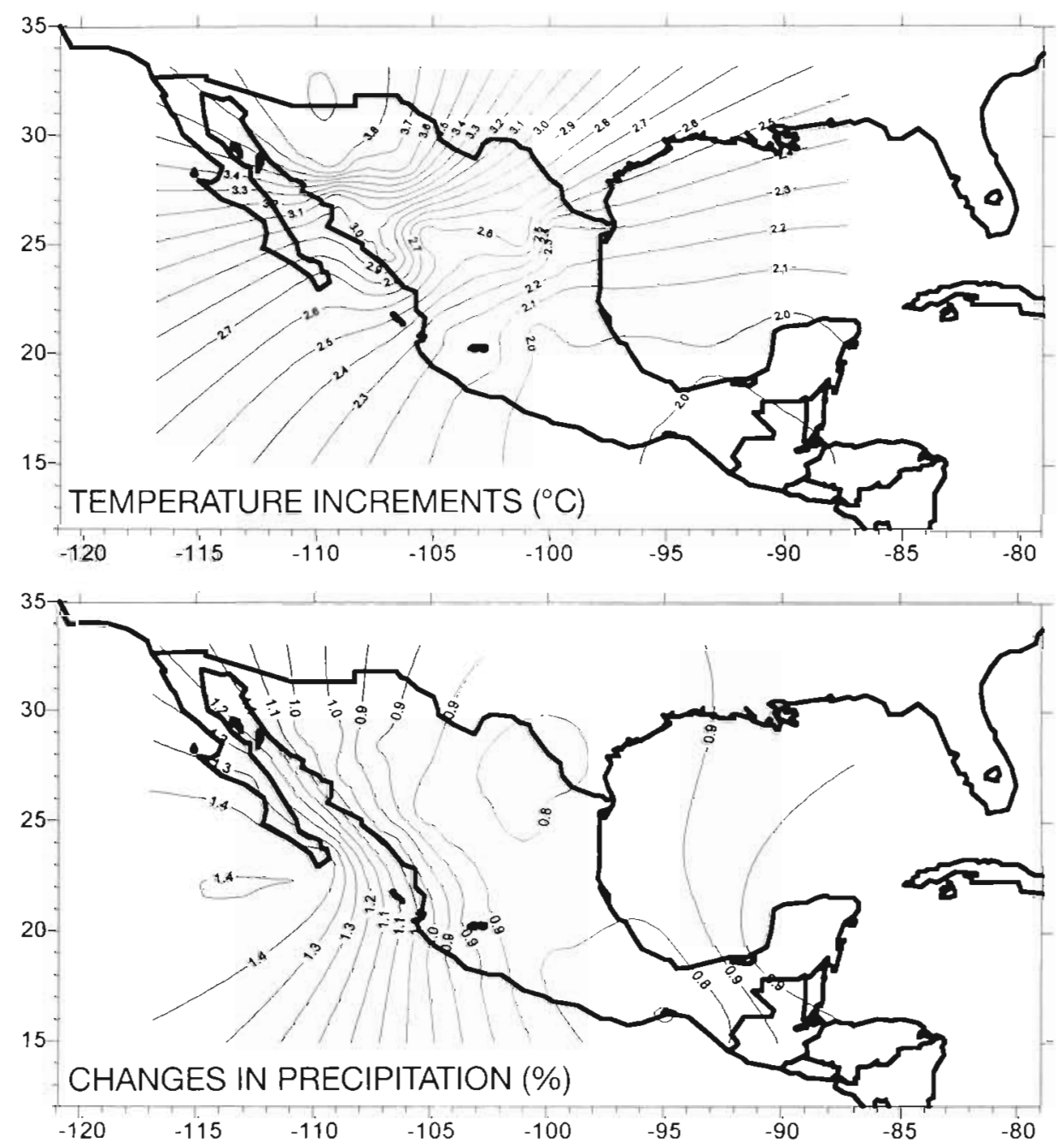

Fig. 5. CCCM-derived summer temperature and precipitation scenarios

latter were: sea level pressure (SLP), sea surface temperature (SST), temperature at 500 and 700 mbar ( 1500 and 700 ), and geopotential altitude at 500 and 700 mbar (z500 and z700) (Magaña 1995).

Regional temperature and precipitation scenarios were developed by defining regression equations between temperature (or precipitation) time series from each one of the 18 Douglas regions (dependent variable) and large-scale SLP and SST patterns at 2 points having the highest correlation with regional temperature or precipitation anomalies (a total of 4 independent variables). The study covered the 1947 to 1987 period.

\section{RESULTS}

The Douglas database includes mean temperature values (92 points) and mean precipitation values (279 points) for a period over 30 yr (1950 to 1980). The 18 regions mentioned above are displayed in Fig. 1, while the isotherms and isohyets produced with 1950 to 1980 data series are shown in Figs. 2 \& 3. This baseline scenario was used in both the agriculture and the desertification and drought vulnerability assessments.

The proposed incremental climate change scenarios were applied at the regional and local levels for the vulnerability analyses of all the above mentioned study sectors in order to assess the sensitivity of each system to arbitrary climate changes.

The data derived from the interpolation of temperature and precipitation variations simulated with the CCCM and GFDL-R30 models were also used for the vulnerability assessments of all study sectors. For regionalization purposes, direct interpolation to points within the 18 regions was carried out. The corresponding potential climate change scenarios were thus derived from the CCCM and GFDL-R30 models. Winter and summer temperature and precipitation maps 

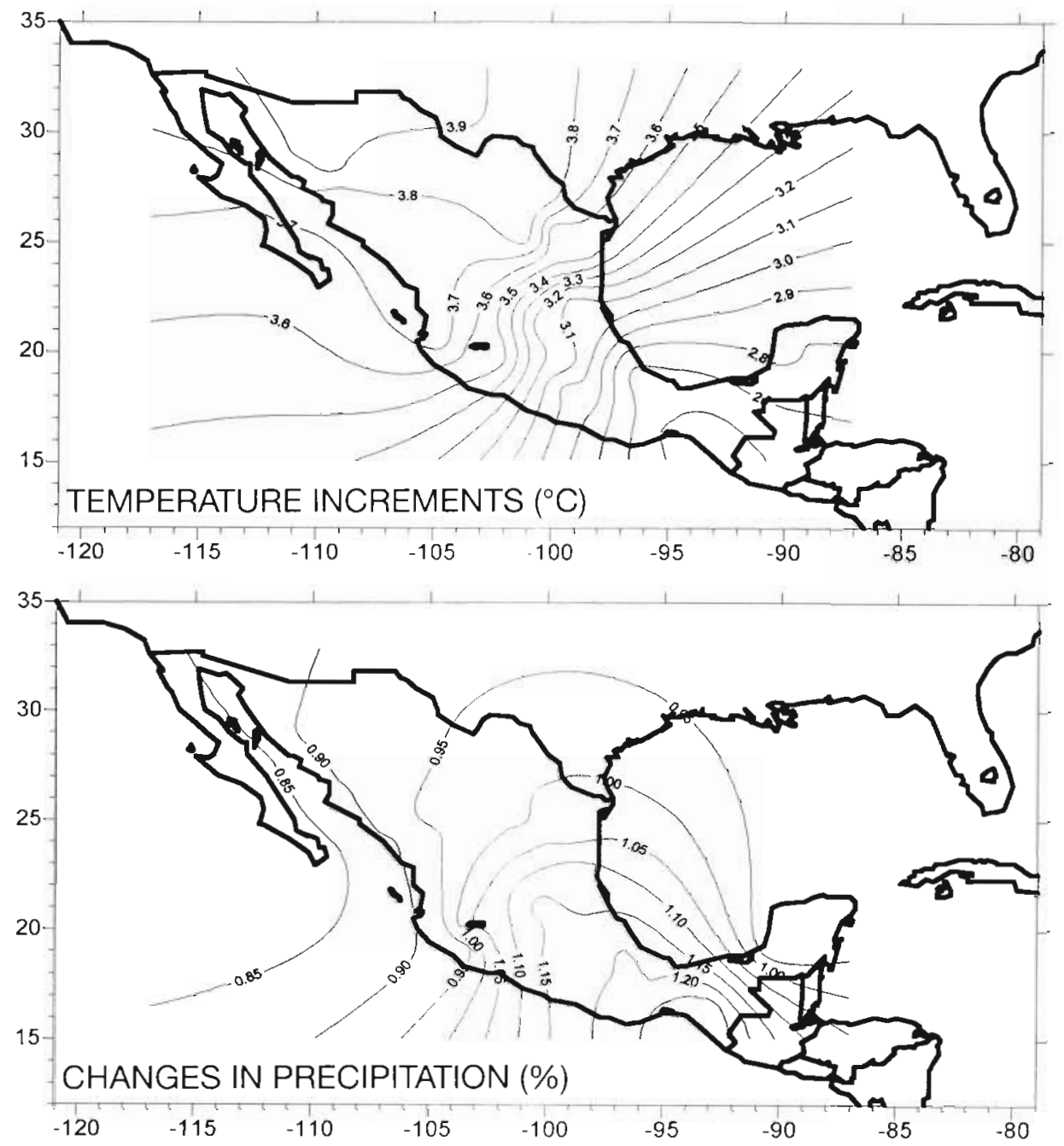

Fig. 6. GFDL-derived winter temperature and precipitation scenarios

were produced based on the CCCM (Figs. 4 \& 5) and GFDL-R30 outputs (Figs. 6 \& 7).

Outputs from both models differ both in magnitude and in sign, as indicated in the figures. High positive changes both in temperature and precipitation are simulated by the GFDL model. The same trend applies for radiation (not shown).

Lower increases in temperature and, in general, decreases in precipitation and radiation were simulated by the CCCM model.

Results of the vulnerability studies will depend on the type of climate change scenario adopted. Thus, similar results to those obtained using the CCCM model scenario were produced when precipitation decreases were considered. In contrast, if a precipitation increase was considered, the results were closer to those produced using the GFDL-R30 scenario.

The vulnerability study sectors did not apply climate scenarios derived from statistical models, which relate current-climate and climate-change large-scale circulation patterns with regional and local climate conditions, because at the first stage of the Country Study project, direct interpolation was the standard methodology for all participant countries. The scenarios produced with that methodology are likely to be used in future vulnerability studies, however.

As an example of the 'downscaling' methodology based on regression equations, the regional temperature anomalies obtained from the $2 \times \mathrm{CO}_{2}-1 \times \mathrm{CO}_{2}$ GCM outputs are displayed in Fig. 8. Lower temperature increments are estimated using this methodology as compared to the direct interpolation method. On the other hand, no difference in the sign of the precipitation anomalies were found between the NCAR and the GFDL outputs. The pattern of the contour lines derived from both models was similar for winter, but was substantially different for the summer, particularly in the northwestern region. 

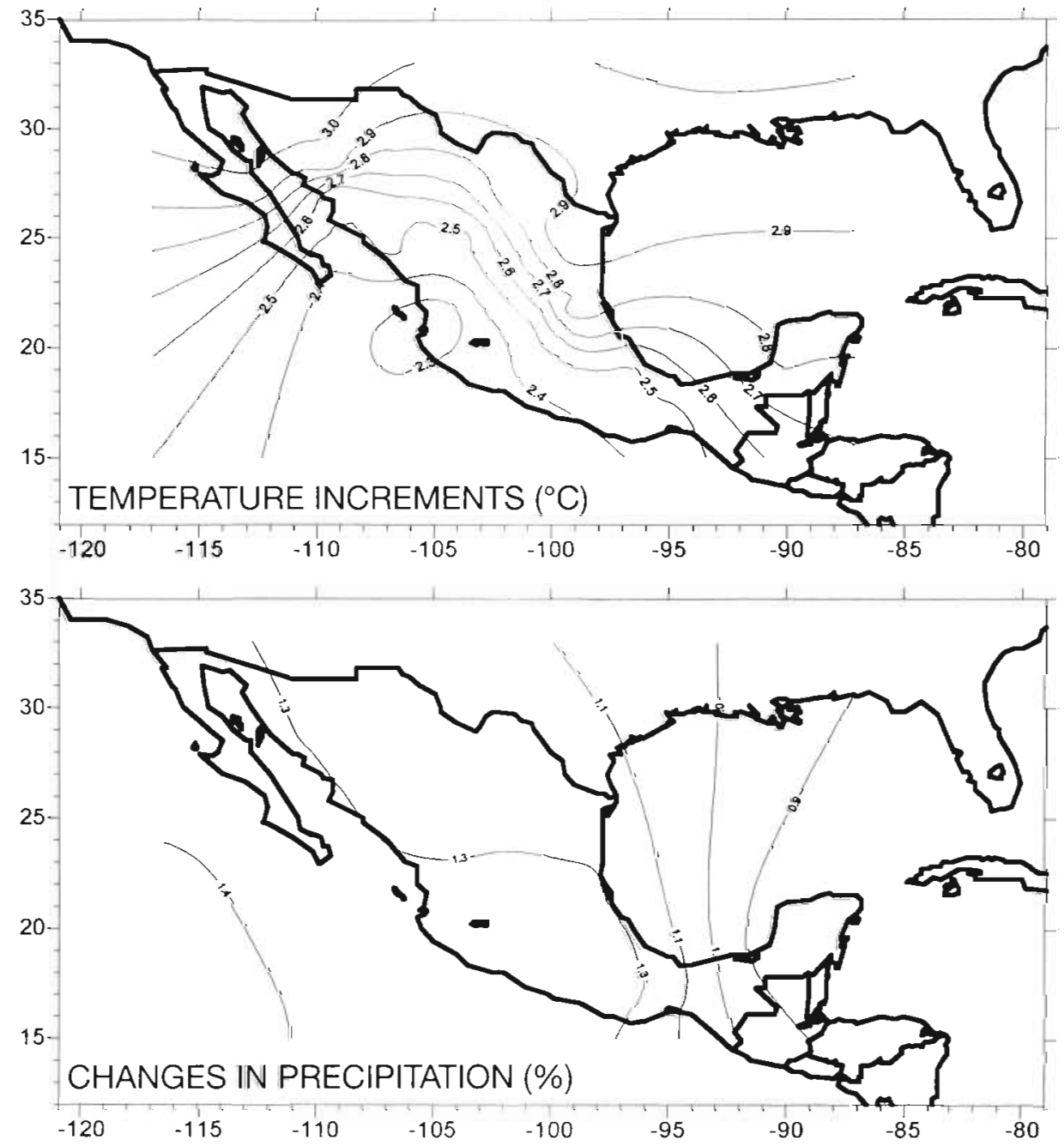

Fig. 7. GFDL-derived summer temperature and precipitation scenarios

\section{DISCUSSION AND CONCLUSIONS}

The regionalization of current climate scenarios for vulnerability studies should be based, first of all, on the identification of the most important areas at the national level for each one of the sectors potentially vulnerable to global climate change (e.g. agriculture, forests). Secondly, regionalization efforts should be made using records from weather stations selected according to homogeneity, altitude and data density criteria for each region.

As regards climate change scenarios, all GCMs simulate, at a large scale, a potential increase in temperature under a condition of doubled atmospheric carbon dioxide concentration and increased emissions of other greenhouse gases. However, the low spatial resolution of the GCMs does not allow for the appropriate simulation of regional and/or local effects, which are largely dependent on factors such as the topography or oceanland interactions. This is a critical problem for the analysis of potential precipitation changes, since the direct interpolation of GCM outputs may result in variations which differ not only in magnitude but also in sign.

The methodology used for regionalization purposes is thus a conclusive factor for global climate change vulnerability studies. In the present study, the correlation of mesoscale variables with large-scale circulation patterns turned out to be a more appropriate method for the development of regional climate change scenarios. Temperature increase values estimated using this method were more moderate than those resulting from the direct interpolation of GCM outputs. Further, practically no differences in sign were obtained for the precipitation variations derived from 2 GCMs. 

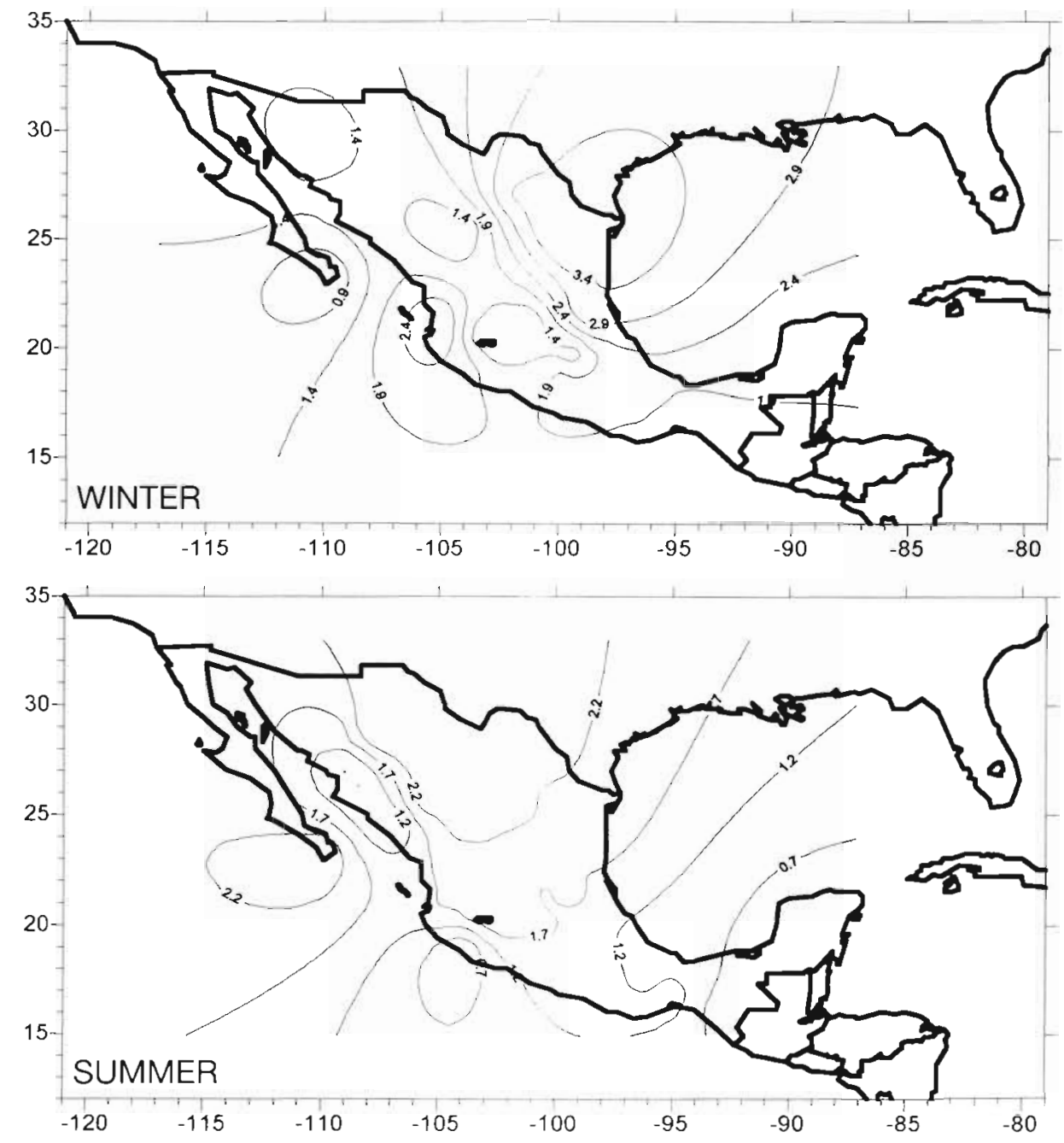

Fig. 8. GFDL-derived $2 \times \mathrm{CO}_{2}-\mathrm{CONTROL}$ temperature anomalies in ${ }^{\circ} \mathrm{C}$. Regionalization according to Douglas

\section{LITERATURE CITED}

Adem J (1982) Simulation of annual cycle of climate with a thermodynamic numerical model. Geof Int 21(3) $229-247$

Conde C, Sánchez O, Gay C (1994) Escenarios básicos y regionales. Estudio de País: México. México ante el cambio climático. Memorias del Primer Taller de Estudio de País: México. Cuernavaca, Mor. México. Instituto Nacional de Ecología and the National Autonomous University of Mexico, Mexico City, p 39-44

Conde C, Sánchez O, Magaña V, Gay C (1995) Escenarios climáticos básicos y regionales. México ante el cambio climático. Memorias del Segundo Taller de Estudio de
País: México. Cuernavaca, Mor. México. Instituto Nacional de Ecología and the National Autonomous University of Mexico, Mexico City, p 101-111

Magana V (1994) A strategy to determine regional climate change. México ante el cambio climático. Memorias del Primer Taller de Estudio de País: México. Cuernavaca, Mor. México. Instituto Nacional de Ecología and the National Autonomous University of Mexico, Mexico City, p 45-51

Magaña V (1995) Escenarios físicos de cambio climático. México ante el cambio climático. Memorias del Segundo Taller de Estudio de País: México. Cuernavaca, Mor. México. Instituto Nacional de Ecología and the National Autonomous University of Mexico, Mexico City, p 93-99 